\title{
Structure and collaboration relationship analysis in a scientific collaboration network
}

\author{
HUI Zi ${ }^{1,2,3^{*}}$, CAI Xu ${ }^{2}$, GRENECHE Jean-Marc ${ }^{3} \&$ WANG Qiuping Alexandre ${ }^{1,3}$ \\ ${ }^{1}$ LUNAM Universite, ISMANS, 44, Ave. Bartholdi, 72000 Le Mans, France; \\ ${ }^{2}$ Complexity Science Center, Institute of Particle Physics, Central China Normal University, Wuhan 430079, China; \\ ${ }^{3}$ LUNAM Universite, Universite du Maine, LPEC, UMR CNRS 6087, 72085 Le Mans, France
}

Received May 17, 2011; accepted July 11, 2011

\begin{abstract}
We construct a weighted network of scientific collaboration in computational geometry and study the statistical properties of the network. In addition, we introduce a parameter called the collaboration relationship parameter to measure the collaboration between scientists. The collaboration relationship parameter of two scientists depends not only on the connection weights between the nodes, but also on the network's structure. The stability of the network's structure in terms of different edge removal strategies is also studied. According to the parameter, we find that a community structure exists in this type of network.
\end{abstract}

scientific collaboration network, phase transition, weighted network, collaboration relationship parameter

Citation: Hui Z, Cai X, Greneche J M, et al. Structure and collaboration relationship analysis in a scientific collaboration network. Chinese Sci Bull, 2011, 56: 3702-3706, doi: 10.1007/s11434-011-4756-9

Many real systems can be mapped onto complex networks, consisting of a set of distinguishable nodes $i=1,2 \ldots N$, and connected by a fixed number of distinguishable edges $l=1$, $2 \ldots N$. These edges represent different relationships between agents, such as friendship, collaboration, business, sexual and other interactions [1-11]. Scientific collaboration is an interesting subject for network research [12-15]. Previous research has uncovered some important results. On one hand, most networks exhibit the small-world property, which means that the network has, at the same time, greater clustering and shorter average shortest path $[1,2,8]$. On the other hand, the degree distribution of a large network follows the power-law distribution $[3-8,10]$. Recently, Newman [12,13] studied several large databases of papers and established some unweighted and undirected collaboration networks, with all the general parts of small-world networks. Li et al. [14,15] constructed a weighted directed network of scientific communication, including collaboration, citation, and personal discussion. A weighted network carries more

*Corresponding author (email: huizi0117@gmail.com) properties than an unweighted one. The way to measure the weight of a link has been introduced differently in various papers [12,13,16-19].

In this paper, we study a scientific collaboration network in computational geometry (the geom network). The static properties of the network, including degree distribution, average clustering coefficient, and average shortest path, are investigated. Furthermore, we are interested in the collaboration relationship between two scientists. To work on the problem, we define the collaboration relationship parameter (CRP) to measure the partnership between two scientists. We observe that the relations with different CRP values have different roles in the network, and that a community structure exists in this network.

\section{Basic statistical result}

The study in this paper is based on the network of Computational Geometry Database from http://vlado.fmf.uni-lj.si/ pub/networks/data/collab/geom.htm. The scientific collabo- 
ration network in computational geometry was produced from a BibTeX bibliography (Beebe, 2002) and obtained from the Computational Geometry Database geombib, version February 2002 (Jones, 2002). In the network, a node represents an author. Two authors are linked by an edge, if they collaborate on a common work (paper, book, etc.). The value of an edge is the number of common works. From the database, we obtained 13919 papers and 7343 authors. The network is described by an adjacency matrix. In this matrix, $a_{i j}=1$ if nodes $i$ and $j$ are linked, and 0 otherwise.

Basic parameters of the network are listed in Table 1. Like other social networks, the clustering coefficient of the network is larger, $\langle c\rangle=0.41$, which means one scientist's coauthors have a higher probability of collaborating with each other. The average shortest path of the network is $<l>=5.31$. The degree distribution [3] of the network follows the power law with an exponent of -2.2 (Figure 1). This scientific collaboration network has the small-world property and a scale-free degree distribution.

As is known, a hierarchical organization is present in some real networks [20]. The clustering coefficient of a node with $k$ edges follows the scaling law in a hierarchical network as given in

$$
c(k) \sim k^{-1} .
$$

From Figure 2, we can see that $c(k)$ scales as $k^{-1}$, indicating that the geom network has a hierarchical organization.

\section{Measure of weight}

In an unweighted network, some important properties are

Table 1 Basic parameters of the scientific collaboration network ${ }^{a)}$

\begin{tabular}{ccccc}
\hline$N$ & $L$ & $<k>$ & $<c>$ & $<l>$ \\
\hline 7343 & 11898 & 3.24 & 0.41 & 5.313 \\
\hline
\end{tabular}

a) $N$ is the number of scientists, $L$ is the number of collaboration relationships, $\langle k>$ is the average degree [3], $\langle c\rangle$ is the mean clustering coefficient [2], and $<l>$ is the average shortest path.

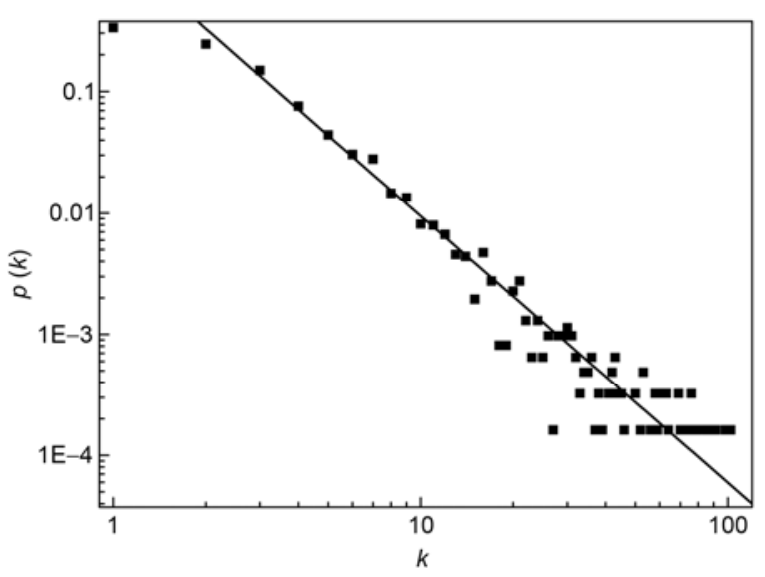

Figure 1 Degree distribution of the network. The solid line has slope $=-2.2$.

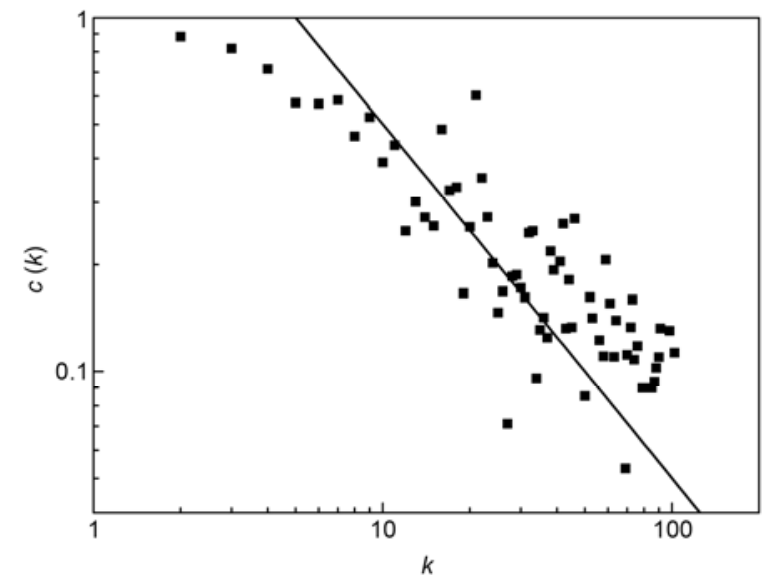

Figure 2 Correlation between the local clustering $c(k)$ and the node's degree $k$. The solid line has slope $=-1$.

ignored. Based on the database, we define two kinds of weights on links and one kind of weight on nodes. One of the link weights is the number of collaborations, which is represented by $w . w_{i j}$ is the number of collaborate works between nodes $i$ and $j$, with $w_{i j}=w_{j i}$. The weight distribution of the network is shown in Figure 3. The distribution of $w$ also follows the power law function with an exponent of -2.4 . The other weight represents the CRP, and is denoted by $o . o_{i j}$ denotes the CRP between nodes $i$ and $j$. The definition of CRP is given as

$$
o_{i j}=\frac{\sum_{k \in G} w_{i j k}+w_{i j}}{m_{i}+m_{j}-w_{i j}-\sum_{k \in G} w_{i j k}},
$$

where $G$ is the set of nodes that are neighbors of either $i$ or $j$ and $m$ is the weight of a node. $m_{i}$ represents the weight of node $i$, defined as $m_{i}=\sum_{i^{\prime}} w_{i i^{\prime}}$, where $i^{\prime}$ denotes all the nodes in the network. $w_{i j k}=\min \left\{w_{i k}, w_{j k}\right\} \times a_{i j}$. CRP is defined as the ratio between the collaborative works of $i$ and

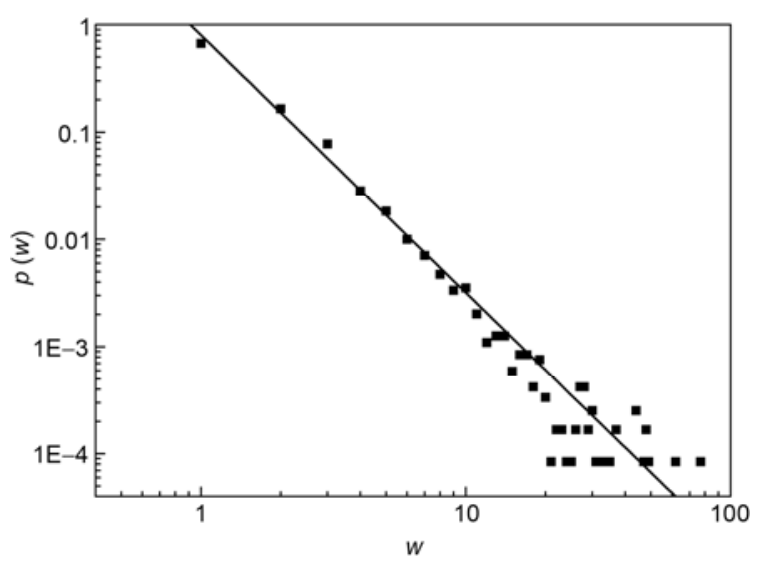

Figure 3 Distribution of the collaboration frequency between two scientists. The solid line has slope $=-2.4$. 
$j$ and the collaborative works of $i$ or $j$. The collaborative works of $i$ and $j$ consist of two components. One is $w_{i j}$, the number of collaborative works between them. The other is $w_{i j k}$, the number of collaborative works on which $i$ and $j$ collaborate with their common neighbors. CRP changes from 0 to $1 . o_{i j}=0$, while nodes $i$ and $j$ are disconnected. The larger $o_{i j}$ is, the closer is the relationship between $i$ and $j$. From the definition, we have $o_{i j}=o_{j i}$. Besides a scientific collaboration network, CRP can be used in other kinds of weighted networks that represent the relationships between nodes, such as an airline network, Facebook network, and so on.

From eq. (2), the CRP of two scientists is concerned not only with the collaborate frequency between them, but also with their neighbors. Two links that have the same $w$ in different network structures, may have different CRP values. In one case, if $i$ and $j$ are only coauthors for each other, the collaboration relationship between them is close, as shown by $o_{i j}=1$ in Figure 4(a). In another case, if $i$ has another coauthor besides $j$, the collaboration relationship between $i$ and $j$ is affected by the cooperation around them, which is shown in Figure 4(b) and (c). In Figure 4, $i$ and $j$ have the same $w$ weight, but different $o$ weights, for the different network structures.

\section{Structure of the network}

To reveal the relationship between CRP and the network structure, we researched the network's ability to withstand the removal of links. To investigate the influence of removing links, we measure the relative size of the giant component $R_{\mathrm{gc}}(f)=\frac{N_{\mathrm{gc}}(f)}{N_{\mathrm{gc}}(0)}$, where $f$ is the parameter for removing a link. If we remove the weakest links first, links that satisfy $o_{i j}<f$ are removed. In contrast, we could begin from the strong links, where those with weight $o_{i j}>f$ are removed. When links are removed, the network breaks into a number of components. $N_{\mathrm{gc}}(f)$ is the number of nodes in the giant component, when we remove the links that are greater or smaller than $f . N_{\mathrm{gc}}(0)$ is the number of nodes of the giant component in the original network. In Figure 5(a), we find that removing the smaller edges first leads to the network's sudden disintegration at $f_{\mathrm{c}} \approx 0.17$. Conversely, removing the stronger edges first decreases the network, but does not unexpectedly break it apart (see Figure 5(c)).

Next, we introduce the average cluster size $\tilde{s}(f)=$ $\sum n_{s} s^{2} / N$ to discuss the changes in $R_{\mathrm{gc}}$ [21,22], where $n_{s}$ is the number of components of size $s$ and the sum takes over
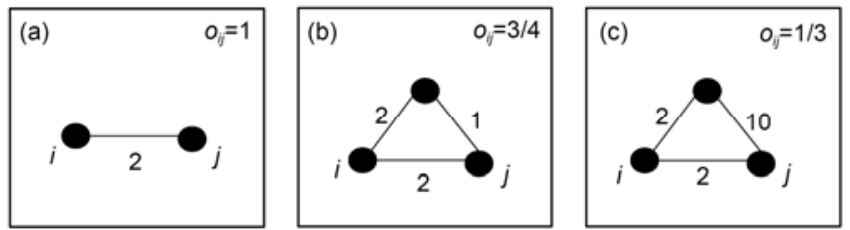

Figure 4 Illustration of the CRP between scientists $i$ and $j$, with values shown for three local network configurations.
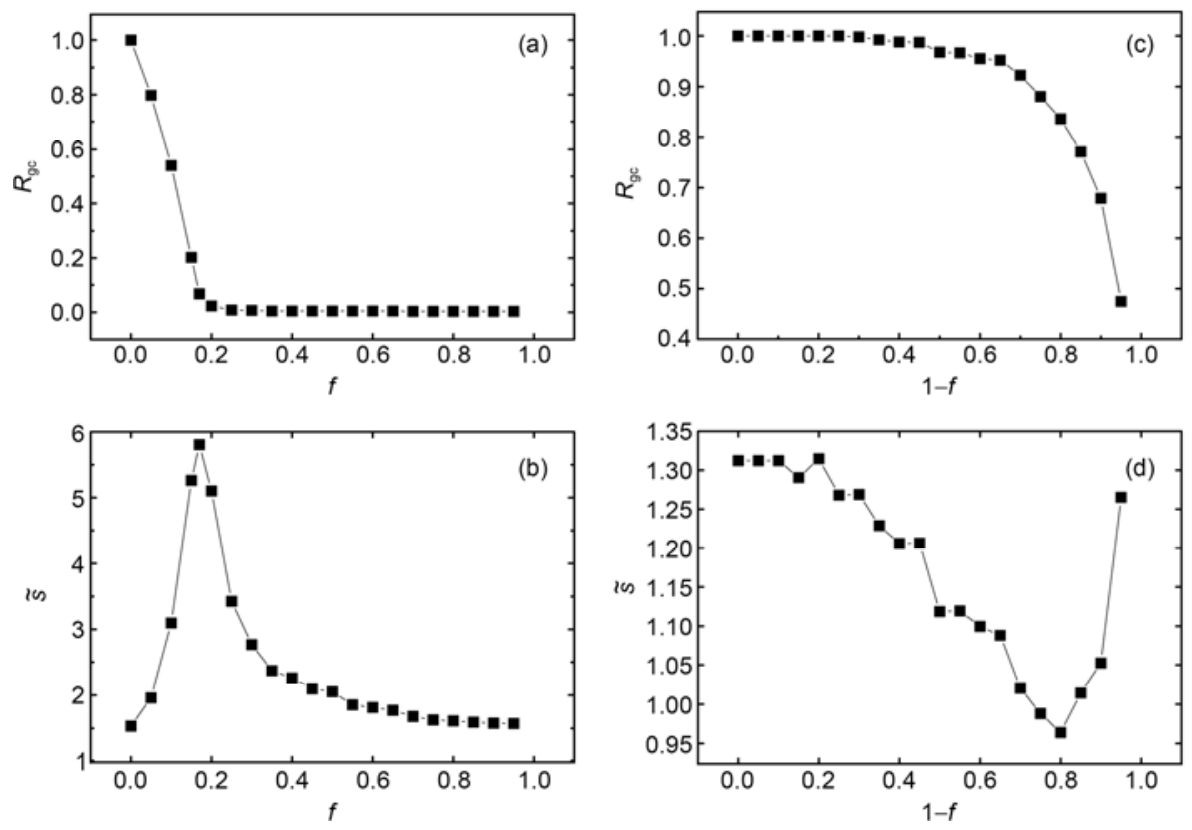

Figure 5 Stability of the scientific collaboration network. The control parameter $f$ denotes the fraction of removed links. (a) and (c) Changes in $R_{\mathrm{gc}}$ when we remove the links, from the weakest or strongest, respectively. (b) and (d) Changes in $\tilde{s}$ when we remove the links, from the weakest or strongest, respectively. 
all but the largest component. $N$ is the number of nodes in the network. According to percolation theory, if the network breaks up because of a phase transition, for $f$ approaching $f_{c}$, $\tilde{s}(f)$ diverges, but in a finite system, the divergence is capped [21,22]. In Figure 5(b), $\tilde{s}(f)$ develops a peak if we remove the smallest links first. In Figure 5(d), when the strongest links are removed first, the peak in $\tilde{s}(f)$ does not occur.

To determine whether the peak in $\tilde{s}(f)$ is a phase transition, we work on the transition $f \rightarrow f_{\mathrm{c}}$. If the network is infinite, $\tilde{s}(f)$ diverges for $f \rightarrow f_{\mathrm{c}}$ as a power law with critical exponent $-\gamma$ in terms of the distance of $f$ from $f_{\mathrm{c}}$ [22], that is, $\tilde{s}(f) \rightarrow\left|f-f_{c}\right|^{-\gamma}$, for $f \rightarrow f_{c}$. In Figure 6, we plot the transformed average cluster size $\log \frac{\tilde{s}(f)}{\tilde{s}_{\max }}$ against $\log \left|f-f_{\mathrm{c}}\right|$ for $f<f_{\mathrm{c}}$ and $f>f_{\mathrm{c}} . \quad \tilde{s}_{\max }$ is the maximum of $\tilde{s}$. The data satisfies $\frac{\tilde{s}(f)}{\tilde{s}_{\max }} \rightarrow\left|f-f_{c}\right|^{-\gamma}$, for $f<f_{\mathrm{c}}$ and $f>f_{\mathrm{c}}$. Therefore the network has a phase transition at $f_{\mathrm{c}} \approx 0.17$, if we remove the smaller links first.

After removing the links from the network, the network is divided into several parts. To measure the structure of the parts, we use the modularity, $Q$ [23], given by

$$
Q=\sum_{i}\left(e_{i i}-a_{i}^{2}\right)
$$

where $e_{i j}$ is the fraction of all edges in the network which link vertices in community $i$ to vertices in community $j$, and $a_{i}=\sum_{j} e_{i j}$ represents the fraction of edges that connect with vertices in community $i$. $Q$ varies between 0 and 1 . A greater $Q$ indicates that the network has a stronger com-

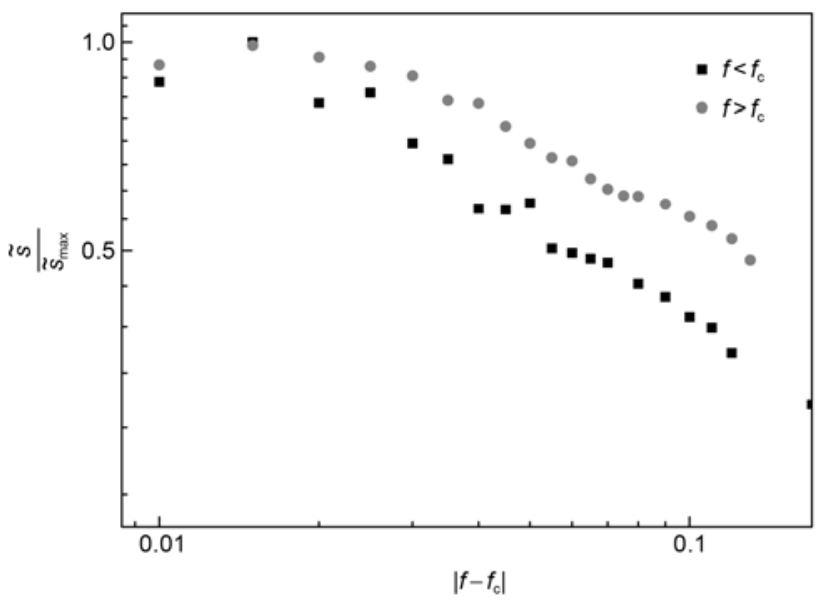

Figure 6 Transformed average cluster size, $\frac{\tilde{s}}{\tilde{s}_{\max }}$, versus $\left|f-f_{\mathrm{c}}\right|$, the distance of $f$ from $f_{\text {c }}$. munity structure.

In Figure 7(a), when $f \approx f_{\mathrm{c}}, Q$ reaches its maximum, which indicates that the network has a strong community structure. If we remove the strongest links first, the peak in $Q$ does not occur, as shown in Figure 7(b). Above all, we find that removing the weakest links first leads the network to break into some small close clusters, but without the strong community structure that exists when the stronger links are removed first.

We can easily conclude from the above discussion that a community structure exists in the geom network. Links with small CRP are used to link dense communities, while those with large CRP exist in the community.

\section{Conclusion}

In this paper, we discussed the topology of a network with the small-world property and power-law degree distribution, similar to some other networks. In addition, we introduced CRP to measure the collaboration relationship between two scientists. The larger the CRP is, the closer is the relationship between two scientists. We found that the removal of links with weak CRP brings about a phase transition similar to the network breaking up, although the removal of strong links has little impact on the network's overall integrity. Next, we revealed that a cluster structure exists in our network. Removing links from the weakest first, when $f \approx f_{\mathrm{c}}$,
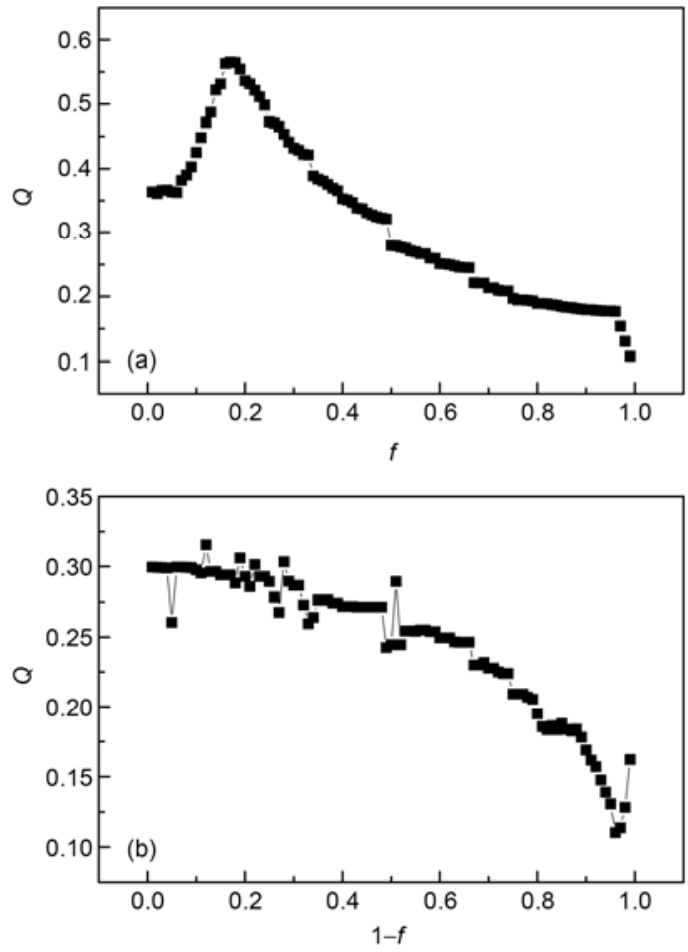

Figure 7 Changes in modularity $Q$ by removing links. (a) If we remove the links from the weakest, $Q$ reaches its maximum at $f \approx f_{\mathrm{c}}$. (b) If the strongest links are removed first, the peak in $Q$ does not occur. 
causes the modularity $Q$ to reach its maximum, which indicates that the geom network has a strong community structure. Links with a weak collaboration relationship connect the small clusters to the large component, while links with a strong collaboration relationship perform the task of reinforcing the clusters.

We would to thank Profs. Li W. and Guo L. for the helpful discussions. This work was supported by the National Natural Science Foundation of China (10647125, 10635020, 10975057 and 10975062), the Program of Introducing Talents of Discipline to Universities (B08033) and the PHC CAI YUAN PEI Program (LIU JIN OU [2010] No. 6050).

1 Watts D J. Small World. Princeton: Princeton University Press, 1999

2 Watts D J, Strogatz S H. Collective dynamics of 'small-world' networks. Nature, 1998, 393: 440-442

3 Barabási A L, Albert R. Emergence of scaling in random networks. Science, 1999, 286: 509-512

4 Zhang P P, Chen K, He Y, et al. Model and empirical study on some collaboration networks. Physica A, 2006, 360: 599-616

5 Chang H, Su B B, Zhou Y P, et al. Assortativity and act degree distribution of some collaboration networks. Physica A, 2007, 383: 687-702

$6 \mathrm{Li} \mathrm{W}$, Cai X. Empirical analysis of a scale-free railway network in China. Physica A, 2007, 382: 693-703

7 Li Y, Wei L X, Niu Y, et al. Structural organization and scale-free properties in Chinese phrase networks. Chinese Sci Bull, 2005, 50: 1304-1308

8 Liu H T. Statistical properties of Chinese semantic networks. Chinese Sci Bull, 2009, 54: 2781-2785

$9 \mathrm{Hu}$ X H, Zhang X Q, Wang H L, et al. Two-port network model and startup criteria for thermoacoustic oscillators. Chinese Sci Bull, 2009, 54: $335-343$

10 Liu X, Liu T Q, Li X Y. A novel evolving model for power grid. Sci China Tech Sci, 2010, 53: 2862-2866

11 Ma J, Wu Y, Ying H P, et al. Channel noise-induced phase transition of spiral wave in networks of Hodgkin-Huxley neurons. Chinese Sci Bull, 2011, 56: 151-157

12 Newman M E J. Scientific collaboration networks. I. Network construction and fundamental results. Phys Rev E, 2001, 64: 016131

13 Newman M E J. Scientific collaboration networks. II. Shortest paths, weighted networks, and centrality. Phys Rev E, 2001, 64: 016132

14 Li M H, Fan Y, Chen J W, et al. Weighted networks of scientific communication: The measurement and geometrical role of weight. Int J Mod Phys B, 2004, 18: 2505

15 Li M H, Fan Y, Chen J W, et al. Weighted networks of scientific communication: The measurement and topological role of weigh. Physica A, 2005, 350: 643-656

16 Macdonald D J, Almaas E, Barabási A L. Minimum spanning trees of weighted scale-free networks. Europhys Lett, 2005, 72: 308

17 Park K, Lai Y C, Ye N. Characterization of weighted complex networks. Phys Rev E, 2004, 70: 026109

18 Onnela J P, Saramaki J, Hyvonen J, et al. Structure and tie strengths in mobile communication networks. Proc Natl Acad Sci USA, 2007, 104: 7332-7336

19 Kumpula J K, Onnela J P, Saramaki J, et al. Emergence of communities in weighted networks. Phys Rev Lett, 2007, 99: 228701

20 Ravasz E, Barabási A L. Hierarchical organization in complex networks. Phys Rev E, 2003, 67: 026112

21 Stauffer D, Ahanony A. Introduction to Percolation Theory. 2nd ed. Boca Raton, FL: CRC Press, 2005

22 Christensen K, Moliney N R. Complexity and Criticality. London: Imperial College Press, 2005

23 Newman M E J, Girvan M. Finding and evaluating community structure in networks. Phys Rev E, 2004, 69: 026113

Open Access This article is distributed under the terms of the Creative Commons Attribution License which permits any use, distribution, and reproduction in any medium, provided the original author(s) and source are credited. 